\title{
FORCING QUASIRANDOMNESS WITH TRIANGLES
}

\author{
CHRISTIAN REIHER and MATHIAS SCHACHT \\ Fachbereich Mathematik, Universität Hamburg, Hamburg, Germany; \\ email: Christian.Reiher@uni-hamburg.de, schacht@math.uni-hamburg.de
}

Received 22 December 2017; accepted 3 March 2019

\begin{abstract}
We study forcing pairs for quasirandom graphs. Chung, Graham, and Wilson initiated the study of families $\mathcal{F}$ of graphs with the property that if a large graph $G$ has approximately homomorphism density $p^{e(F)}$ for some fixed $p \in(0,1]$ for every $F \in \mathcal{F}$, then $G$ is quasirandom with density $p$. Such families $\mathcal{F}$ are said to be forcing. Several forcing families were found over the last three decades and characterizing all bipartite graphs $F$ such that $\left(K_{2}, F\right)$ is a forcing pair is a well-known open problem in the area of quasirandom graphs, which is closely related to Sidorenko's conjecture. In fact, most of the known forcing families involve bipartite graphs only.

We consider forcing pairs containing the triangle $K_{3}$. In particular, we show that if $\left(K_{2}, F\right)$ is a forcing pair, then so is $\left(K_{3}, F^{\triangleright}\right)$, where $F^{\triangleright}$ is obtained from $F$ by replacing every edge of $F$ by a triangle (each of which introduces a new vertex). For the proof we first show that $\left(K_{3}, C_{4}^{\triangleright}\right)$ is a forcing pair, which strengthens related results of Simonovits and Sós and of Conlon et al.
\end{abstract}

2010 Mathematics Subject Classification: 05C80

\section{Introduction}

The systematic study of quasirandom graphs was initiated by Thomason [53, 54] and Chung, Graham, and Wilson [12] and over the last 30 years many generalizations and extensions to directed graphs [23], tournaments [7], hypergraphs [1, 4-6, 16, 26, 34, 36, 55], set systems [8], permutations [19], groups [22], subsets of cyclic groups and finite fields [10, 56], and sparse graphs $[11,15,32]$ were established by several researchers (see, for example, the surveys $[33,35]$ for a more detailed discussion). Roughly speaking, a given discrete structure is quasirandom if it shares important properties with a 'truly random' structure of the same size. In the context of graphs this is made precise by mimicking the uniform edge distribution of the random graph $G(n, p)$.

(C) The Author(s) 2019. This is an Open Access article, distributed under the terms of the Creative Commons Attribution licence (http://creativecommons.org/licenses/by/4.0/), which permits unrestricted re-use, distribution, and reproduction in any medium, provided the original work is properly cited. 
Definition 1.1. For $\varepsilon>0$ and $p \in(0,1]$ we say a graph $G=(V, E)$ is $(\varepsilon, p)$ quasirandom, if for all subsets $X, Y \subseteq V$ we have

$$
\left|e_{G}(X, Y)-p\right| X|| Y|| \leqslant \varepsilon|V|^{2},
$$

where edges contained in the intersection $X \cap Y$ are counted twice in $e_{G}(X, Y)$.

In the context of quasirandom graphs we often consider sequences of graphs $\vec{G}=\left(G_{n}\right)_{n \in \mathbb{N}}$ with $\left|V\left(G_{n}\right)\right| \rightarrow \infty$. Then we may say that the sequence $\vec{G}$ is $p$-quasirandom if for every $\varepsilon>0$ all but finitely many members of the sequence are $(\varepsilon, p)$-quasirandom. For a simpler discussion, we sometimes say that a graph $G$ is $p$-quasirandom without any reference to the sequence, by which we mean that some sufficiently large graph $G$ is $(\varepsilon, p)$-quasirandom for some small unspecified $\varepsilon>0$. If the density $p$ is of no particular importance, then we may just say $G$ is quasirandom.

A large part of the theory of quasirandom graphs concerns equivalent characterizations of $p$-quasirandom graph sequences. Early results in that direction implicitly appeared in $[2,3,20,41]$ and Chung, Graham, and Wilson [12] gave six alternative characterizations. Since then many more such characterizations were found (see, for example, [9, 16, 28, 30, 42-44, 47-50, 57]).

Here we focus on characterizations that rely on the densities of graph homomorphisms of given graphs $F$ into large graphs $G$. We denote by $\operatorname{hom}(F, G)$ the number of graph homomorphisms from $F$ into $G$ and the homomorphism density $t(F, G)$ is defined by

$$
t(F, G)=\frac{\operatorname{hom}(F, G)}{|V(G)|^{|V(F)|}} .
$$

Let us recall that a pair of graphs $\left(F_{1}, F_{2}\right)$ is said to be forcing if for every $p \in(0,1]$ and $\varepsilon>0$ there is some $\delta>0$ such that the following holds: if a graph $G$ satisfies

$$
t\left(F_{1}, G\right) \geqslant(1-\delta) p^{e\left(F_{1}\right)} \quad \text { and } \quad t\left(F_{2}, G\right) \leqslant(1+\delta) p^{e\left(F_{2}\right)}
$$

then it is $(\varepsilon, p)$-quasirandom. This notion goes back to [12] and has frequently been discussed in the literature. The most classical example of such a family is the pair $\left(K_{2}, C_{4}\right)$. The statement that the pair $\left(K_{2}, F\right)$ is forcing for every bipartite graph $F$ that is not a forest, called the forcing conjecture, can be traced back to Skokan and Thoma [50] (see also [14]). It has been the subject of intensive study that led to its verification in various cases. For the most recent contributions to the forcing conjecture and the closely related conjectures of Erdôs and Simonovits [46] and of Sidorenko [45] we refer to [14, 17, 18, 25, 31, 37, 51]. 
Until recently all known forcing pairs contained bipartite graphs only. In fact, already Chung, Graham, and Wilson [12] noted that $\left(K_{2}, K_{3}\right)$ (and also $\left(K_{1,2}, K_{3}\right)$ ) is not a forcing pair, by giving an example of $n$-vertex graphs $G$ with all vertices having degree close to $n / 2$ and with $t\left(K_{3}, G\right) \approx 1 / 8$, but containing independent sets and cliques of size $\lfloor n / 4\rfloor$. However, it was shown by Simonovits and Sós [48] that such a situation can be avoided by appealing to the hereditary nature of quasirandom graphs, that is, if $G=(V, E)$ is $p$-quasirandom, then the induced subgraphs $G[U]$ are $p$-quasirandom for linear sized subsets $U \subseteq V$. Simonovits and Sós then showed that requiring

$$
t(F, G[U])=\left(1 \pm \delta \frac{|V|}{|U|}\right) p^{e(F)}
$$

for a given graph $F$ with at least one edge and for all $U \subseteq V$ forces $G$ to be $p$-quasirandom. Recently, Conlon, Hàn, Person, and Schacht [16] (see also [24]) observed that condition (1.2) gives rise to a forcing pair $\left(F, M_{F}\right)$ for an appropriate graph $M_{F}$ depending on $F$.

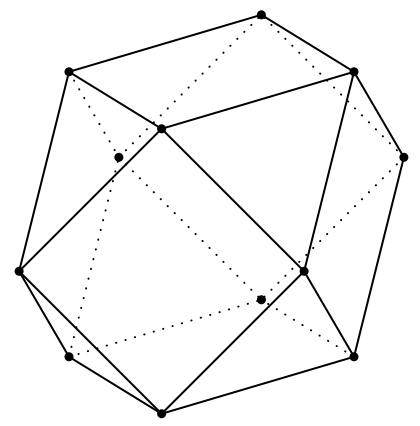

We study forcing pairs involving triangles. For that case it was shown in [16] that the pair $\left(K_{3}, M\right)$ is forcing, where $M$ denotes the line graph of the 3-dimensional Boolean cube, depicted above. The idea behind the proof is roughly as follows: Three successive applications of the Cauchy-Schwarz inequality yield $t(M, G) \geqslant t\left(K_{3}, G\right)^{8}$ for any graph $G=(V, E)$. On the other hand, assuming (1.1) for $\left(K_{3}, M\right)$ tells us $t\left(K_{3}, G\right) \gtrsim p^{3}$ and $t(M, G) \lesssim p^{24}$ for some real $p \in(0,1]$. Consequently, an approximate equality must hold in each of these three steps, and it may be argued that this is in turn only possible if for all subsets $A, B, C \subseteq V$ we have

$$
\triangle(A, B, C) \approx p^{3}|A||B||C|,
$$

where $\triangle(A, B, C)$ denotes the number of triangles with a vertex in $A$, a vertex in $B$, and a vertex in $C$. This yields (1.2) for $F=K_{3}$ and the Simonovits-Sós theorem implies that $G$ is $p$-quasirandom. 
Our main result shows that the same effect as above can be achieved with two applications of the Cauchy-Schwarz inequality only. This implies that the pair $\left(K_{3}, C_{4}^{\triangleright}\right)$ is forcing, where $C_{4}^{\triangleright}$ is obtained from the 4-cycle $C_{4}$ where every edge is replaced by a triangle (each of which introduces a new vertex), that is, the graph shown below. As we shall explain in more detail below, we have $t\left(C_{4}^{\triangleright}, G\right) \geqslant t\left(K_{3}, G\right)^{4}$ for all graphs $G$, and if approximate equality holds for some graph $G$, then it satisfies the assumption of the following theorem, which weakens the assumption of the Simonovits-Sós theorem in the triangle case.

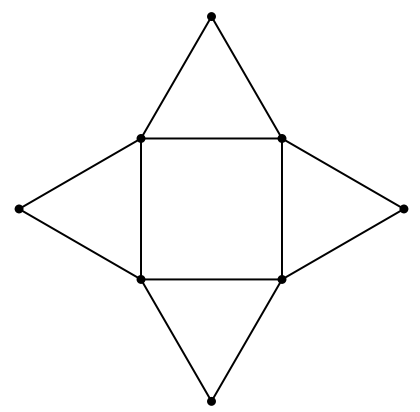

THEOREM 1.2. For every $p \in(0,1]$ and $\varepsilon>0$ there is an $\eta>0$ such that any graph $G=(V, E)$ satisfying

$$
\triangle(A, B, V)=p^{3}|A||B||V| \pm \eta|V|^{3}
$$

for all $A, B \subseteq V$ is $(\varepsilon, p)$-quasirandom.

The following corollary renders the aforementioned connection between condition (1.3) and $t\left(C_{4}^{\triangleright}, G\right) \approx t\left(K_{3}, G\right)^{4}$ and strengthens the result of Conlon et al. that $\left(K_{3}, M\right)$ is a forcing pair.

COROLlARY 1.3. The pair $\left(K_{3}, C_{4}^{\triangleright}\right)$ is forcing.

As it turns out, Corollary 1.3 applied in the context of weighted graphs allows the following general result, which is our main result on forcing pairs involving triangles.

Corollary 1.4. If $\left(K_{2}, F\right)$ is a forcing pair, then so is $\left(K_{3}, F^{\triangleright}\right)$.

Organization. We prove Theorem 1.2 in Section 2 and Corollary 1.3 in Section 3. In Section 4, we switch to the analytical language of graphons and 
prove Corollary 1.4. We conclude by recording some further observations and problems for future research in Section 5.

\section{The two sets condition}

The proof of Theorem 1.2 is based on the regularity method for graphs. This means that we use a regularity lemma and a counting lemma in order to reduce the problem at hand to a somewhat different one that speaks about a certain 'reduced graph'. In the present situation we need to conceive this reduced graph as a weighted graph. Such objects may also be regarded as symmetric matrices with entries from the unit interval. The precise statement we shall require is stated as Lemma 2.1 below. The interested reader may check that this lemma could conversely also be deduced from Theorem 1.2.

LEMMA 2.1. Given any real numbers $p \in(0,1]$ and $\varepsilon>0$ there is a real $\delta>0$ such that the following holds: let $\left(d_{i j}\right)_{i, j \in[t]} \in[0,1]^{t \times t}$ be a symmetric matrix such that for all distinct indices $i, j \in[t]$ we have

$$
d_{i j} \sum_{k \in[t]} d_{i k} d_{j k}=\left(p^{3} \pm \delta\right) t
$$

Then $d_{i j}=p \pm \varepsilon$ holds for all $i, j \in[t]$.

Proof. Throughout the proof we work with the hierarchy $\delta \ll \varrho \ll p$, $\varepsilon$ for some auxiliary chosen constant $\varrho$, where we write $\alpha \ll \beta$ to signify that $\alpha$ will be chosen sufficiently small depending on $\beta$.

Since the sum on the left-hand side of equation (2.1) is at most $t$, we have $d_{i j} \geqslant p^{3}-\delta \geqslant p^{3} / 2$ for all $i, j \in[t]$. The main idea is to choose three indices $x$, $y, z \in[t]$ so that the difference $d_{x y}-d_{x z}$ is as large as possible. In the picture to the bottom, these indices $x, y$, and $z$ are represented as 'vertices' and the labels $a$ and $b$ attached to the 'edges' $x y$ and $x z$ indicate that we set $a=d_{x y}$ and $b=d_{x z}$, respectively. The vertex $w$, which is shown there as well, will be chosen later in the argument.




The maximality of $a-b$ entails

$$
d_{y i} \geqslant b \quad \text { and } \quad d_{z i} \leqslant a \quad \text { for all } i \in[t] .
$$

By (2.1) we have

$$
a \cdot \sum_{i \in[t]} d_{x i} d_{y i} \leqslant\left(p^{3}+\delta\right) t \quad \text { and } \quad b \cdot \sum_{i \in[t]} d_{x i} d_{z i} \geqslant\left(p^{3}-\delta\right) t,
$$

whence

$$
\sum_{i \in[t]} d_{x i}\left(a d_{y i}-b d_{z i}\right) \leqslant 2 \delta t
$$

Now (2.2) yields

$$
a d_{y i}-b d_{z i}=a\left(d_{y i}-b\right)+b\left(a-d_{z i}\right) \geqslant\left(\left(d_{y i}-b\right)+\left(a-d_{z i}\right)\right) p^{3} / 2 \geqslant 0
$$

for all $i \in[t]$, so it follows that

$$
\sum_{i \in[t]}\left(d_{y i}-b\right)+\sum_{i \in[t]}\left(a-d_{z i}\right) \leqslant 8 p^{-6} \delta t
$$

where neither sum on the left-hand side is negative. Thus there is an index $w \in[t]$ with $\left(d_{w y}-b\right)+\left(a-d_{w z}\right) \leqslant 8 p^{-6} \delta$. In particular, the numbers $\bar{a}=d_{w z} \leqslant a$ and $\bar{b}=d_{w y} \geqslant b($ see $(2.2))$ satisfy

$$
|a-\bar{a}|+|\bar{b}-b| \leqslant 8 p^{-6} \delta .
$$

Applying (2.1) to the pairs $(w, y)$ and $(w, z)$ in place of $(i, j)$ and subtracting the resulting estimates we obtain

$$
\left|\sum_{i \in[t]} d_{w i}\left(\bar{a} d_{z i}-\bar{b} d_{y i}\right)\right| \leqslant 2 \delta t .
$$

Thus the triangle inequality and (2.3) lead to

$$
\begin{aligned}
|a \bar{a}-b \bar{b}| \cdot p^{3} t / 2 \leqslant & \left|\sum_{i \in[t]} d_{w i}(a \bar{a}-b \bar{b})\right| \\
\leqslant & \left|\sum_{i \in[t]} d_{w i} \bar{a}\left(a-d_{z i}\right)\right| \\
& +\left|\sum_{i \in[t]} d_{w i} \bar{b}\left(d_{y i}-b\right)\right|+\left|\sum_{i \in[t]} d_{w i}\left(\bar{a} d_{z i}-\bar{b} d_{y i}\right)\right| \\
\leqslant & 2\left(1+4 p^{-6}\right) \delta t,
\end{aligned}
$$


that is, $|a \bar{a}-b \bar{b}| \leqslant 4 p^{-3}\left(1+4 p^{-6}\right) \delta$. Consequently (2.4) yields

$p^{3}(a-b) \leqslant a^{2}-b^{2} \leqslant|a \bar{a}-b \bar{b}|+a|a-\bar{a}|+b|\bar{b}-b| \leqslant 4 p^{-3}\left(1+4 p^{-6}\right) \delta+8 p^{-6} \delta$ and thus $a-b \leqslant 4 p^{-6}\left(1+2 p^{-3}+4 p^{-6}\right) \delta \leqslant \varrho$.

Now for any four indices $i, j, k, \ell \in[t]$ the extremal choice of $a-b$ gives

$$
\left|d_{i j}-d_{k \ell}\right| \leqslant\left|d_{i j}-d_{i \ell}\right|+\left|d_{i \ell}-d_{k \ell}\right| \leqslant 2(a-b) \leqslant 2 \varrho .
$$

In other words, there is an interval of length $2 \varrho$ containing all the $d_{i j}$. In the light of (2.1) and the smallness of $\varrho$ this interval needs to be contained in $(p-\varepsilon, p+\varepsilon)$. Thereby Lemma 2.1 is proved.

As we have already said, our proof of Theorem 1.2 depends on Szemerédi's regularity lemma [52], a version of which we would like to state next.

THEOREM 2.2 (Regularity lemma). For every positive real number $\delta$ there is a positive integer $T$ such that every graph $G=(V, E)$ admits a partition $V=V_{0} \bullet V_{1} \bullet \cdots \bullet V_{t}$ of its vertex set obeying the following conditions:

(a) $t \leqslant T,\left|V_{0}\right| \leqslant \delta|V|$, and $\left|V_{1}\right|=\cdots=\left|V_{t}\right|>0$.

(b) For each $i \in[t]$ there are at most $\delta t$ many indices $j \in[t]$ such that the pair $\left(V_{i}, V_{j}\right)$ is not $\delta$-quasirandom.

Here a pair $(A, B)$ of nonempty subsets of $V$ with density $d=e(A, B) /|A||B|$ is said to be $\delta$-quasirandom if we have $e(X, Y)=d|X||Y| \pm \delta|A||B|$ for all $X \subseteq A$ and $Y \subseteq B$.

The above statement differs in several aspects from the 'standard' regularity lemma and we briefly discuss those differences:

- The crucial property obtained for most pairs $\left(V_{i}, V_{j}\right)$ is often taken to be something called $\delta$-regularity, where a pair $(A, B)$ of subsets of $G$ is said to be $\delta$-regular if $|d(X, Y)-d(A, B)| \leqslant \delta$ holds for all $X \subseteq A$ and $Y \subseteq B$ with $|X| \geqslant \delta|A|$ and $|Y| \geqslant \delta|B|$, rather than $\delta$-quasirandomness. These two concepts are known to be equivalent up to polynomial losses in the involved constants, and in fact $\delta$-regularity implies $\delta$-quasirandomness. Our reason for working with this notion here is that allows a slightly cleaner presentation of the proof.

- Instead of the second condition one usually finds a weaker clause just stating that at most $\delta t^{2}$ pairs $\left(V_{i}, V_{j}\right)$ fail to be quasirandom in the literature. The above version has also been used and to obtain it, one may apply the standard version of the regularity lemma with some appropriate $\delta^{\prime} \ll \delta$ in place of $\delta$ and then relocate all classes $V_{i}$ with $i>0$ that violate (b) to $V_{0}$. 
- Usually one requires also a lower bound $t_{0}$ on the number of vertex classes $t$ in advance and then one obtains $T \geqslant t \geqslant \min \left(t_{0},|V|\right)$ rather than just $T \geqslant t$ in the first part of (a). The rationale behind this is that in many applications one has no intentions of 'looking inside the individual $V_{i}$ ', wherefore it brings certain advantages to have these sets reasonably small. In our current situation, however, even the extreme outcome $t=1$ would be useful. In view of (b) it would mean that the pair $\left(V_{1}, V_{1}\right)$ is $\delta$-quasirandom, and since, provided that $\delta$ is small, $V_{1}$ would be almost all of $V(G)$; this is essentially all we need to infer for the proof of Theorem 1.2.

In the course of proving Theorem 1.2 we will also need to be able to count triangles after regularizing $G$. This will be rendered by the following strong, but well-known, form of the triangle counting lemma.

Lemma 2.3 (Triangle counting lemma). Let $A, B$, and $C$ denote three nonempty subsets of $V(G)$ for some graph $G$. Suppose that the pairs $(B, C),(C, A)$, and $(A, B)$ have edge densities $\alpha, \beta$, and $\gamma$, respectively, and that at least two of these three pairs are $\delta$-quasirandom. Then $\triangle(A, B, C)=(\alpha \beta \gamma \pm 2 \delta)|A||B||C|$.

We apply Lemma 2.1 together with the regularity method in form of Theorem 2.2 and Lemma 2.3 and deduce Theorem 1.2.

Proof of Theorem 1.2. We begin by choosing certain auxiliary constants obeying the hierarchy

$$
\eta \ll T^{-1} \ll \delta \ll \varepsilon, p,
$$

where $T$ is the integer obtained by applying the regularity Theorem 2.2 with $\delta$. Now let any graph $G=(V, E)$ satisfying

$$
\triangle(A, B, V)=p^{3}|A||B||V| \pm \eta|V|^{3}
$$

for all $A, B \subseteq V$ be given. The regularity lemma yields a partition

$$
V(G)=V_{0} \bullet V_{1} \bullet \cdots \bullet V_{t}
$$

satisfying the above clauses (a) and (b). For $i, j \in[t]$ we denote the density of the pair $\left(V_{i}, V_{j}\right)$ by $d_{i j}$. The assumption (2.5) is only going to be used in the special case $A, B \in\left\{V_{1}, \ldots, V_{t}\right\}$. It then discloses the following useful property of the numbers $d_{i j}$ :

$$
d_{i j} \sum_{k \in[t]} d_{i k} d_{j k}=\left(p^{3} \pm 9 \delta\right) t,
$$

for all $i, j \in[t]$. To see this, we consider any two indices $i, j \in[t]$. Let $R_{i}$ denote the set of all $k \in[t]$ for which the pair $\left(V_{i}, V_{k}\right)$ is not $\delta$-quasirandom, let $R_{j}$ be 
defined similarly with respect to $j$, and set $R=R_{i} \cup R_{j}$. Owing to condition (b) from Theorem 2.2 we have $\left|R_{i}\right| \leqslant \delta t$ and $\left|R_{j}\right| \leqslant \delta t$, whence $|R| \leqslant 2 \delta t$. Let us write $M=\left|V_{1}\right|=\cdots=\left|V_{t}\right|$. Then $M t=|V|-\left|V_{0}\right| \geqslant(1-\delta)|V|$. As we may assume $\delta \leqslant \frac{1}{2}$, it follows that $|V| \leqslant 2 M t$, whence $\left|V_{0}\right| \leqslant 2 \delta M t$. Now we have

$$
\begin{aligned}
& \left|\Delta\left(V_{i}, V_{j}, V\right)-M^{3} d_{i j} \sum_{k \in[t]} d_{i k} d_{j k}\right| \\
& \quad \leqslant \triangle\left(V_{i}, V_{j}, V_{0}\right)+\sum_{k \in[t]}\left|\triangle\left(V_{i}, V_{j}, V_{k}\right)-M^{3} d_{i j} d_{i k} d_{j k}\right| .
\end{aligned}
$$

Here the first term may be estimated trivially by

$$
\triangle\left(V_{i}, V_{j}, V_{0}\right) \leqslant\left|V_{0}\right|\left|V_{i}\right|\left|V_{j}\right| \leqslant 2 \delta M^{3} t .
$$

Moreover, for $k \in[t] \backslash R$ the triangle counting lemma (Lemma 2.3) tells us that

$$
\left|\triangle\left(V_{i}, V_{j}, V_{k}\right)-M^{3} d_{i j} d_{i k} d_{j k}\right| \leqslant 2 \delta M^{3},
$$

while for $k \in R$ we still have the obvious bound

$$
\left|\triangle\left(V_{i}, V_{j}, V_{k}\right)-M^{3} d_{i j} d_{i k} d_{j k}\right| \leqslant M^{3} .
$$

Due to $|R| \leqslant 2 \delta t$ all this combines to

$$
\left|\triangle\left(V_{i}, V_{j}, V\right)-M^{3} d_{i j} \sum_{k \in[t]} d_{i k} d_{j k}\right| \leqslant 6 \delta M^{3} t .
$$

On the other hand, plugging $A=V_{i}$ and $B=V_{j}$ into (2.5) we learn

$$
\left|\triangle\left(V_{i}, V_{j}, V\right)-p^{3} M^{2}\right| V|| \leqslant \eta|V|^{3},
$$

which in turn yields

$$
\left|\triangle\left(V_{i}, V_{j}, V\right)-p^{3} M^{3} t\right| \leqslant p^{3} M^{2}(|V|-M t)+\eta|V|^{3} .
$$

In view of

$$
\begin{aligned}
M^{2}(|V|-M t)+\eta|V|^{3} & =M^{2}\left|V_{0}\right|+\eta|V|^{3} \\
& \leqslant M^{3}\left(2 \delta t+8 \eta t^{3}\right) \leqslant M^{3} t\left(2 \delta+8 \eta T^{2}\right)
\end{aligned}
$$

a suitable choice of $\eta$ leads to

$$
\left|\triangle\left(V_{i}, V_{j}, V\right)-p^{3} M^{3} t\right| \leqslant 3 \delta M^{3} t .
$$

Together with (2.7) this concludes the proof of (2.6). 
We may assume that depending on $\varepsilon$ and $p$ the constant $\delta$ has been chosen so small that Lemma 2.1 guarantees $d_{i j}=p \pm \varepsilon / 2$ for all $i, j \in[t]$. Let us write $S$ for the set of all pairs $(i, j) \in[t]^{2}$ such that the pair $\left(V_{i}, V_{j}\right)$ is not $\delta$-quasirandom. Notice that condition (b) from Theorem 2.2 implies $|S| \leqslant \delta t^{2}$.

Now for any $A, B \subseteq V$ we have

$$
|e(A, B)-p| A|| B|| \leqslant \sum_{i=0}^{t} \sum_{j=0}^{t}\left|e\left(A \cap V_{i}, B \cap V_{j}\right)\right|-p\left|A \cap V_{i}\right|\left|B \cap V_{j}\right| \mid .
$$

Each term on the left-hand side having $i=0, j=0$, or $(i, j) \in S$ may be bounded from above by $\left|V_{i}\right|\left|V_{j}\right|$, so altogether these terms contribute at most

$$
|V|^{2}-\left(|V|-\left|V_{0}\right|\right)^{2}+|S| M^{2} \leqslant 2 \delta|V|^{2}+\delta M^{2} t^{2} \leqslant 3 \delta|V|^{2} .
$$

Owing to the quasirandomness, each of the remaining terms on the right-hand side of (2.8) may be estimated as follows:

$$
\begin{aligned}
&\left|e\left(A \cap V_{i}, B \cap V_{j}\right)-p\right| A \cap V_{i}|| B \cap V_{j}|| \\
& \leqslant\left|e\left(A \cap V_{i}, B \cap V_{j}\right)-d_{i j}\right| A \cap V_{i}|| B \cap V_{j}|| \\
&+\left|d_{i j}-p\right|\left|V_{i}\right|\left|V_{j}\right| \\
& \leqslant\left(\delta+\frac{\varepsilon}{2}\right)\left|V_{i}\right|\left|V_{j}\right| .
\end{aligned}
$$

So taken together these terms amount to at most $(\delta+\varepsilon / 2)|V|^{2}$, and in view of $\delta \ll \varepsilon$ we finally arrive at

$$
|e(A, B)-p| A|| B|| \leqslant\left(4 \delta+\frac{\varepsilon}{2}\right)|V|^{2} \leqslant \varepsilon\left|V^{2}\right|
$$

for arbitrary $A, B \subseteq V$. This proves that $G$ is $(\varepsilon, p)$-quasirandom.

\section{Proof of Corollary 1.3}

In this section, we deduce Corollary 1.3. The only thing we need to check is the following proposition, which combined with Theorem 1.2 yields the corollary.

Proposition 3.1. Suppose that a graph $G=(V, E)$ satisfies

$$
t\left(K_{3}, G\right) \geqslant(1-\delta) p^{3} \quad \text { and } t\left(C_{4}^{\triangleright}, G\right) \leqslant(1+\delta) p^{12}
$$

for some $p, \delta \in(0,1]$. Then

$$
\triangle(A, B, V)=p^{3}|A||B||V| \pm 8 \delta^{1 / 4} p^{3}|V|^{3}
$$

holds for all $A, B \subseteq V$. 
Besides the Cauchy-Schwarz inequality itself the proof will also use the following known and easy to confirm result on situations where equality almost holds.

FACT 3.2. Let $x_{1}, \ldots, x_{n}, \alpha$, and $v$ denote any real numbers satisfying

$$
\sum_{i=1}^{n} x_{i}=\alpha n \quad \text { and } \quad \sum_{i=1}^{n} x_{i}^{2}=\left(\alpha^{2}+v^{2}\right) n .
$$

Then we have $\sum_{i=1}^{m} x_{i}=\alpha m \pm v n$ for any $m=0,1, \ldots, n$.

Proof of Proposition 3.1. Let $G=(V, E), p$, and $\delta$ be as in the hypothesis and let $A, B \subseteq V$ be arbitrary.

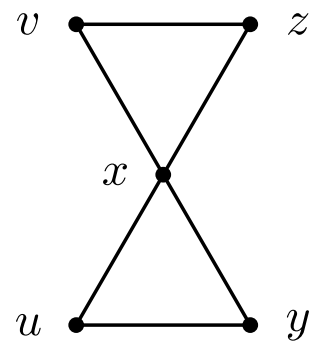

We begin the proof by setting up some notation: Let $S$ denote the graph obtained by gluing two triangles together at a vertex (see the picture depicted above). We consider the homomorphism densities of $K_{3}, S$, and $C_{4}^{\triangleright}$ and define the real numbers $a, b$, and $c$ so as to obey

$$
\begin{aligned}
\operatorname{hom}\left(K_{3}, G\right) & =a p^{3} n^{3}, \\
\operatorname{hom}(S, G) & =b p^{6} n^{5},
\end{aligned}
$$

and

$$
\operatorname{hom}\left(C_{4}^{\triangleright}, G\right)=c p^{12} n^{8},
$$

where $n=|V|$. Notice that the assumption translates to $a \geqslant 1-\delta$ and $c \leqslant 1+\delta$. Given a vertex $x \in V$ we write $T_{x}$ for the number of pairs $(y, z) \in V^{2}$ such that $x y z$ is a triangle in $G$. Moreover, for any two vertices $u, v \in V$ we denote the number of triples $(x, y, z) \in V^{3}$ with $u x, u y, v x, v z, x y, x z \in E$ by $S_{u, v}$ (see figure). In terms of these numbers the equations (3.1), (3.2), and (3.3) rewrite as

$$
\sum_{x \in V} T_{x}=a p^{3} n^{3}
$$




$$
\sum_{x \in V} T_{x}^{2}=\sum_{(u, v) \in V^{2}} S_{u, v}=b p^{6} n^{5}
$$

and

$$
\sum_{(u, v) \in V^{2}} S_{u, v}^{2}=c p^{12} n^{8}
$$

Thus the Cauchy-Schwarz inequality implies

$$
(1-\delta)^{4} \leqslant a^{4} \leqslant b^{2} \leqslant c \leqslant 1+\delta .
$$

Due to Fact 3.2 (applied with $\alpha=a p^{3} n^{2}$ and $v=p^{3} n^{2} \sqrt{b-a^{2}}$ ), (3.4), and (3.5) we have

$$
\triangle(A, V, V)=\sum_{x \in A} T_{x}=a p^{3} n^{2}|A| \pm \sqrt{b-a^{2}} \cdot p^{3} n^{3}
$$

Owing to $1-\delta \leqslant a \leqslant 1+\delta$ and $\sqrt{b-a^{2}} \leqslant 3 \sqrt{\delta}$ (see (3.7)), this leads to

$$
\triangle(A, V, V)=p^{3} n^{2}|A| \pm 4 \delta^{1 / 2} p^{3} n^{3} .
$$

Similarly Fact 3.2 (applied with $\alpha=b p^{6} n^{3}$ and $v=p^{6} n^{3} \sqrt{c-b^{2}}$ ), (3.5), and (3.6) give

$$
\sum_{(u, v) \in A^{2}} S_{u, v} \leqslant b p^{6} n^{3}|A|^{2}+\sqrt{c-b^{2}} \cdot p^{6} n^{5} \leqslant\left((1+\delta)|A|^{2}+3 \delta^{1 / 2} n^{2}\right) p^{6} n^{3},
$$

whence

$$
\sum_{y \in V} \triangle(A, y, V)^{2}=\sum_{(u, v) \in A^{2}} S_{u, v} \leqslant\left(|A|^{2}+4 \delta^{1 / 2} n^{2}\right) p^{6} n^{3}
$$

Now if $Q$ and $R$ denote the real numbers satisfying

$$
\sum_{y \in V} \triangle(A, y, V)=p^{3} n^{2} Q \quad \text { and } \quad \sum_{y \in V} \triangle(A, y, V)^{2}=p^{6} n^{3} R
$$

then (3.8) and (3.9) entail

$$
Q=|A| \pm 4 \delta^{1 / 2} n \quad \text { and } \quad R \leqslant|A|^{2}+4 \delta^{1 / 2} n^{2}
$$

whilst a final application of Fact 3.2 (applied with $\alpha=p^{3} n Q$ and $v=p^{3} n \sqrt{R-Q^{2}}$ ) reveals

$$
\triangle(A, B, V)=p^{3} n Q|B| \pm p^{3} n^{2} \sqrt{R-Q^{2}} .
$$


It follows from (3.10) that $R-Q^{2} \leqslant 12 \delta^{1 / 2} n^{2}$ and owing to (3.11) we obtain

$$
\begin{aligned}
\left|\triangle(A, B, V)-p^{3}\right| A|| B|n| & \leqslant p^{3} n|B||Q-| A||+4 \delta^{1 / 4} p^{3} n^{3} \\
& \leqslant p^{3} n^{3}\left(4 \delta^{1 / 2}+4 \delta^{1 / 4}\right) \\
& \leqslant 8 \delta^{1 / 4} p^{3} n^{3}
\end{aligned}
$$

as desired.

\section{Proof of Corollary 1.4}

4.1. Notation. We mostly follow the notation from Lovász's research monograph [38] and in this subsection we remind the reader of what we actually need. By $\mathcal{W}$ we mean the space of all bounded symmetric measurable functions from the unit square $[0,1]^{2}$ to the set of reals. So $\mathcal{W}$ is a linear space whose members are sometimes referred to as kernels. It is known that for each kernel $W$ the maximum

$$
\|W\|_{\square}=\max \left\{\left|\int_{A \times B} W(x, y) d x d y\right|: A, B \subseteq[0,1] \text { measurable }\right\}
$$

exists and that $W \longmapsto\|W\|_{\square}$ is a norm on $\mathcal{W}$, the so-called cutnorm. If $\|W\|_{\square}=0$ holds for $W \in \mathcal{W}$, then this kernel vanishes almost everywhere, that is, the set $\left\{(x, y) \in[0,1]^{2}: W(x, y) \neq 0\right\}$ has measure zero (see [38, Section 8.2.3]).

The group of measure preserving bijections form the unit interval onto itself is denoted by $\mathcal{S}_{[0,1]}$. This group acts in an obvious way on the space of kernels by

$$
W^{\varphi}(x, y)=W(\varphi(x), \varphi(y))
$$

for all $W \in \mathcal{W}, \varphi \in \mathcal{S}_{[0,1]}$, and $x, y \in[0,1]$. The cut distance $\delta_{\square}\left(W_{1}, W_{2}\right)$ between two kernels $W_{1}, W_{2} \in \mathcal{W}$ is defined by

$$
\delta_{\square}\left(W_{1}, W_{2}\right)=\inf \left\{\left\|W_{1}-W_{2}^{\varphi}\right\|_{\square}: \varphi \in \mathcal{S}_{[0,1]}\right\} .
$$

Actually, this infimum is known to be a minimum, but this fact is rarely needed and we shall make no use of it.

Those $W \in \mathcal{W}$ that satisfy $W(x, y) \in[0,1]$ for all $x, y \in[0,1]$ are called graphons and the set of all graphons is denoted by $\mathcal{W}_{0}$. With each graph $G=(V, E)$ we can associate a graphon $W_{G}$ by taking an arbitrary partition $[0,1]=\bigcup_{v \in V} P_{v}$ of the unit interval into measurable pieces of measure $|V|^{-1}$ and defining for all $x, y \in[0,1]$

$$
W_{G}(x, y)= \begin{cases}1, & \text { if } u v \in E, \text { where } x \in P_{u}, y \in P_{v}, \\ 0, & \text { else. }\end{cases}
$$


This graphon depends, of course, not only on $G$ but also on the underlying partition, but modulo the action of $\mathcal{S}_{[0,1]}$ mentioned above it is uniquely determined by $G$.

An important insight due to Lovász and Szegedy [39] is the compactness of the pseudometric space $\left(\mathcal{W}_{0}, \delta_{\square}\right)$. In fact, the compactness easily implies the regularity lemma for graphs (see also [38, Theorem 9.23]). This result does actually occupy a central place in the limit theory of dense graphs. Besides, it is beautifully complemented by the fact that the set $\left\{W_{G}: G\right.$ is a graph $\}$ is dense in $\left(\mathcal{W}_{0}, \delta_{\square}\right)$.

Given a graph $F$ and a kernel $W$ the homomorphism density $t(F, W)$ is defined to be the multidimensional integral

$$
t(F, W)=\int_{[0,1]^{V(F)}} \prod_{u v \in E(F)} W\left(x_{u}, x_{v}\right) \prod_{u \in V(F)} d x_{u} .
$$

This stipulation extends the usual definition of homomorphism densities for graphs in the sense that $t\left(F, W_{G}\right)=t(F, G)$ holds for all graphs $F$ and $G$.

Analytically speaking, the global counting lemma asserts that for every graph $F$ the map $W \longmapsto t(F, W)$ from $\left(\mathcal{W}_{0}, \delta_{\square}\right)$ to $[0,1]$ is Lipschitz continuous with Lipschitz constant $e(F)$ (see [38, Theorem 10.23]).

4.2. Forcing families. Let us write $W \equiv p$ for a kernel $W$ and a real number $p$ if $W$ agrees almost everywhere with the constant function whose value is always $p$.

LEMMA 4.1. A pair of graphs $\left(F_{1}, F_{2}\right)$ is forcing if and only if we have $W \equiv p$ for every real $p \in(0,1]$ and every graphon $W$ with $t\left(F_{i}, W\right)=p^{e\left(F_{i}\right)}$ for $i=1,2$.

Proof. This is implicit in the discussion from [38, Section 16.7.1].

Theorem 1.2 is the discrete analogue of the following statement.

THEOREM 4.2. If a graphon $W$ and a real $p \in(0,1]$ are such that

$$
W(x, y) \int_{0}^{1} W(x, z) W(y, z) d z=p^{3}
$$

holds for almost all $(x, y) \in[0,1]^{2}$, then $W \equiv p$.

One way to show this proceeds by carefully repeating the proof of Lemma 2.1 in this analytical setting. This is not hard, but somewhat technical, and hence we would like to present an alternative argument here. 
Proof of Theorem 4.2. Define a graphon $U$ by

$$
U(x, y)=W(x, y) \int_{0}^{1} W(x, z) W(y, z) d z
$$

for all $x, y \in[0,1]$. Now the assumption $U \equiv p^{3}$ leads to

$$
t\left(K_{3}, W\right)=t\left(K_{2}, U\right)=p^{3} \quad \text { and } \quad t\left(C_{4}^{\triangleright}, W\right)=t\left(C_{4}, U\right)=p^{12} .
$$

Since the pair $\left(K_{3}, C_{4}^{\triangleright}\right)$ is forcing, it follows by Lemma 4.1 that we have indeed $W \equiv p$.

We conclude this section with the proof of our main result on forcing pairs involving triangles.

Proof of Corollary 1.4. Suppose that $W$ is a graphon and $p \in(0,1]$ is a real number such that

$$
t\left(K_{3}, W\right)=p^{3} \quad \text { and } \quad t\left(F^{\triangleright}, W\right)=p^{3 e(F)} .
$$

In view of Lemma 4.1 we have to prove that $W \equiv p$. To this end, we look again at the graphon $U$ defined by (4.1). The hypothesis (4.2) rewrites in terms of $U$ as

$$
t\left(K_{2}, U\right)=p^{3} \quad \text { and } \quad t(F, U)=p^{3 e(F)} .
$$

Since the pair $\left(K_{2}, F\right)$ is forcing, it follows that $U \equiv p^{3}$, and in the light of Theorem 4.2 we get indeed $W \equiv p$.

\section{Concluding Remarks}

We close with a few remarks and open problems for future research.

- Corollary 1.4 raises the general problem to characterize all graphs $F$ with the property that the pair $\left(K_{3}, F\right)$ is forcing. However, given our current state of knowledge and the fact that this is still open for $\left(K_{2}, F\right)$, it appears unclear how to even formulate a plausible conjecture in this regard.

- The proof of Theorem 1.2 presented in Section 2 is based on Szemerédi's regularity lemma and as a consequence this proof requires that $\eta^{-1}$ behaves like an exponential tower of height poly $\left(\varepsilon^{-1}, p^{-1}\right)$. We would like to thank L. M. Lovász for pointing out to us that a different argument utilizing the Frieze-Kannan regularity lemma [21] shows that $\eta^{-1}=2^{(\varepsilon p)^{-\Theta(1)}}$ would suffice as well. To see this one exploits that the assumption of Theorem 1.2 implies $t\left(K_{3}, G\right) \approx p^{3}$ and $t\left(C_{4}^{\triangleright}, G\right) \approx p^{12}$. Due to the global counting lemma 
this gives us two approximate equalities for the densities $d_{i j}$ arising in a Frieze-Kannan regular partition of $G$. As in the proof of Proposition 3.1 two reverse applications of the Cauchy-Schwarz inequality then entail that the assumption (2.1) of Lemma 2.1 holds with at most $o\left(t^{2}\right)$ exceptions. Working a little bit harder in the proof of this lemma it can then be shown that this is enough to imply that there are at most $o\left(t^{2}\right)$ pairs $(i, j) \in[t]^{2}$ for which $d_{i j} \approx p$ fails. This, however, is in turn equivalent to $G$ being $p$-quasirandom.

Recently, it was shown by Conlon, Fox, and Sudakov [13] that the corresponding dependency of the parameters in the Simonovits-Sós theorem for the triangle is in fact linear (see also [27, 40] for further results). In view of these results, it seems an interesting open question whether Theorem 1.2 holds also for $\eta=\operatorname{poly}(\varepsilon, p)$.

- It appears to be an intriguing open problem to find the appropriate generalization of Theorem 1.2 (and Corollary 1.3) for graphs other than the triangle. At this point even for cliques $K_{k}$ with $k \geqslant 4$ this is an open problem. For integers $1 \leqslant \ell \leqslant k$ we say $K_{k}$ is $\ell$-forcing, if every graph $G=(V, E)$ satisfying for all subsets $X_{1}, \ldots, X_{\ell} \subseteq V$

$$
\mathcal{K}_{k}\left(X_{1}, \ldots, X_{\ell}\right)=p^{\left(\begin{array}{l}
k \\
2
\end{array}\right)|V|^{k-\ell}} \prod_{i=1}^{\ell}\left|X_{i}\right|+o\left(|V|^{k}\right)
$$

for some $p \in(0,1]$ is $p$-quasirandom, where $\mathcal{K}_{k}\left(X_{1}, \ldots, X_{\ell}\right)$ denotes the number of $k$-tuples

$$
\left(v_{1}, \ldots, v_{k}\right) \in X_{1} \times \cdots \times X_{\ell} \times V^{k-\ell}
$$

that span a $K_{k}$ in $G$. The Simonovits-Sós theorem implies for every $k \geqslant 2$ that $K_{k}$ is $k$-forcing and it is not hard to show that no clique is 1 -forcing. Theorem 1.2 tells us that $K_{3}$ is 2 -forcing and it would be interesting to determine for every $k \geqslant 4$ the smallest $\ell$ such that $K_{k}$ is $\ell$-forcing. The proof of Theorem 1.2 can be adjusted to show that $\ell=\lceil(k+1) / 2\rceil$ suffices and this was also noted independently by Hubai et al. [29]. Currently, we are not aware of any reason that rules out the possibility that every clique $K_{k}$ is 2-forcing or that there is a universal bound independent of $k$.

- One may also consider hypergraphs extensions of those results. For example, one may investigate, whether the tetrahedron $K_{4}^{(3)}$ is 3 -forcing for the notion of quasirandomness investigated in $[6,34]$.

\section{Acknowledgements}

The research of both author was supported by ERC Consolidator Grant 724903. 


\section{References}

[1] E. Aigner-Horev, D. Conlon, H. Hàn, Y. Person and M. Schacht, 'Quasirandomness in hypergraphs', Electron. J. Combin. 25(3) (2018), Paper 3.34, 22 pages.

[2] N. Alon, 'Eigenvalues and expanders', Combinatorica 6(2) (1986), 83-96. Theory of Computing (Singer Island, Fla., 1984).

[3] N. Alon and F. R. K. Chung, 'Explicit construction of linear sized tolerant networks', in Proceedings of the First Japan Conference on Graph Theory and Applications (Hakone, 1986), Discrete Mathematics, 72 (Elsevier, North-Holland, 1988), 15-19.

[4] F. R. K. Chung, 'Quasi-random classes of hypergraphs', Random Structures Algorithms 1(4) (1990), 363-382.

[5] F. R. K. Chung, 'Quasi-random hypergraphs revisited', Random Structures Algorithms 40(1) (2012), 39-48.

[6] F. R. K. Chung and R. L. Graham, 'Quasi-random hypergraphs', Random Structures Algorithms 1(1) (1990), 105-124.

[7] F. R. K. Chung and R. L. Graham, 'Quasi-random tournaments', J. Graph Theory 15(2) (1991), 173-198.

[8] F. R. K. Chung and R. L. Graham, 'Quasi-random set systems', J. Amer. Math. Soc. 4(1) (1991), 151-196.

[9] F. R. K. Chung and R. L. Graham, 'Maximum cuts and quasirandom graphs', in Random Graphs, vol. 2 (Poznań, 1989) (Wiley-Intersci. Publ., Wiley, New York, 1992), 23-33.

[10] F. R. K. Chung and R. L. Graham, 'Quasi-random subsets of $Z_{n}$ ', J. Combin. Theory Ser. A 61(1) (1992), 64-86.

[11] F. R. K. Chung and R. L. Graham, 'Sparse quasi-random graphs', Combinatorica 22(2) (2002), 217-244, Special issue: Paul Erdős and his mathematics.

[12] F. R. K. Chung, R. L. Graham and R. M. Wilson, 'Quasi-random graphs', Combinatorica 9(4) (1989), 345-362.

[13] D. Conlon, J. Fox and B. Sudakov, 'Hereditary quasirandomness without regularity', Math. Proc. Cambridge Philos. Soc. 164(3) (2018), 385-399.

[14] D. Conlon, J. Fox and B. Sudakov, 'An approximate version of Sidorenko's conjecture', Geom. Funct. Anal. 20(6) (2010), 1354-1366.

[15] D. Conlon, J. Fox and Y. Zhao, 'Extremal results in sparse pseudorandom graphs', Adv. Math. 256 (2014), 206-290.

[16] D. Conlon, H. Hàn, Y. Person and M. Schacht, 'Weak quasi-randomness for uniform hypergraphs', Random Structures Algorithms 40(1) (2012), 1-38.

[17] D. Conlon, J. H. Kim, C. Lee and J. Lee, 'Some advances on Sidorenko's conjecture', J. Lond. Math. Soc. (2) 98(3) (2018), 593-608.

[18] D. Conlon and J. Lee, 'Finite reflection groups and graph norms', Adv. Math. 315 (2017), 130-165.

[19] J. N. Cooper, 'Quasirandom permutations', J. Combin. Theory Ser. A 106(1) (2004), 123-143.

[20] P. Frankl, V. Rödl and R. M. Wilson, 'The number of submatrices of a given type in a Hadamard matrix and related results', J. Combin. Theory Ser. B 44(3) (1988), 317-328.

[21] A. Frieze and R. Kannan, 'Quick approximation to matrices and applications', Combinatorica 19(2) (1999), 175-220.

[22] W. T. Gowers, 'Quasirandom groups', Combin. Probab. Comput. 17(3) (2008), 363-387.

[23] S. Griffiths, 'Quasi-random oriented graphs', J. Graph Theory 74(2) (2013), 198-209.

[24] H. Hàn, Y. Person and M. Schacht, 'Note on forcing pairs', in The Sixth European Conference on Combinatorics, Graph Theory and Applications (EuroComb 2011), Electronic Notes in Discrete Mathematics, 38 (Elsevier Science B. V., Amsterdam, 2011), 437-442. 
[25] H. Hatami, 'Graph norms and Sidorenko's conjecture', Israel J. Math. 175 (2010), 125-150.

[26] J. Haviland and A. Thomason, 'Pseudo-random hypergraphs', Discrete Math. 75(1-3) (1989), 255-278, Graph Theory and Combinatorics (Cambridge, 1988).

[27] X. He, 'Linear dependence between hereditary quasirandomness conditions', Electron. J. Combin. 25(4) (2018), Paper 4.12, 14 pages.

[28] H. Huang and C. Lee, 'Quasi-randomness of graph balanced cut properties', Random Structures Algorithms 41(1) (2012), 124-145.

[29] T. Hubai, D. Král, O. Parczyk and Y. Person, 'More non-bipartite forcing pairs', submitted.

[30] S. Janson and V. T. Sós, 'More on quasi-random graphs, subgraph counts and graph limits', European J. Combin. 46 (2015), 134-160.

[31] J. H. Kim, C. Lee and J. Lee, 'Two approaches to Sidorenko's conjecture', Trans. Amer. Math. Soc. 368(7) (2016), 5057-5074.

[32] Y. Kohayakawa and V. Rödl, 'Regular pairs in sparse random graphs. I', Random Structures Algorithms 22(4) (2003), 359-434.

[33] Y. Kohayakawa and V. Rödl, 'Szemerédi's regularity lemma and quasi-randomness', in Recent advances in algorithms and combinatorics, CMS Books Math./Ouvrages Math. SMC, 11 (Springer, New York, 2003), 289-351.

[34] Y. Kohayakawa, V. Rödl and J. Skokan, 'Hypergraphs, quasi-randomness, and conditions for regularity', J. Combin. Theory Ser. A 97(2) (2002), 307-352.

[35] M. Krivelevich and B. Sudakov, 'Pseudo-random graphs', in More Sets, Graphs and Numbers, Bolyai Society Mathematical Studies, 15 (Springer, Berlin, 2006), 199-262.

[36] J. Lenz and D. Mubayi, 'Eigenvalues and linear quasirandom hypergraphs', Forum Math. Sigma 3(e2) (2015), 26.

[37] J. X. Li and B. Szegedy, 'On the logarithmic calculus and Sidorenko's conjecture', Combinatorica to appear, Preprint, arXiv:1107.1153.

[38] L. Lovász, Large Networks and Graph Limits, American Mathematical Society Colloquium Publications, 60 (American Mathematical Society, Providence, RI, 2012), xiv+475.

[39] L. Lovász and B. Szegedy, 'Szemerédi's lemma for the analyst', Geom. Funct. Anal. 17(1) (2007), 252-270.

[40] C. Reiher and M. Schacht, 'Quasirandomness from hereditary subgraph densities'. Manuscript.

[41] V. Rödl, 'On universality of graphs with uniformly distributed edges', Discrete Math. 59(1-2) (1986), 125-134.

[42] A. Shapira, 'Quasi-randomness and the distribution of copies of a fixed graph', Combinatorica 28(6) (2008), 735-745.

[43] A. Shapira and R. Yuster, 'The effect of induced subgraphs on quasi-randomness', Random Structures Algorithms 36(1) (2010), 90-109.

[44] A. Shapira and R. Yuster, 'The quasi-randomness of hypergraph cut properties', Random Structures Algorithms 40(1) (2012), 105-131.

[45] A. F. Sidorenko, 'Inequalities for functionals generated by bipartite graphs', Diskret. Mat. 3(3) (1991), 50-65. (Russian); English transl., Discrete Math. Appl. 2(5) (1992) 489-504.

[46] M. Simonovits, 'Extremal graph problems, degenerate extremal problems, and supersaturated graphs', in Progress in Graph Theory (Waterloo, Ont., 1982) (Academic Press, Toronto, ON, 1984), 419-437.

[47] M. Simonovits and V. T. Sós, 'Szemerédi's partition and quasirandomness', Random Structures Algorithms 2(1) (1991), 1-10.

[48] M. Simonovits and V. T. Sós, 'Hereditarily extended properties, quasi-random graphs and not necessarily induced subgraphs', Combinatorica 17(4) (1997), 577-596. 
[49] M. Simonovits and V. T. Sós, 'Hereditary extended properties, quasi-random graphs and induced subgraphs', Combin. Probab. Comput. 12(3) (2003), 319-344, Combinatorics, Probability and Computing (Oberwolfach, 2001).

[50] J. Skokan and L. Thoma, 'Bipartite subgraphs and quasi-randomness', Graphs Combin. 20(2) (2004), 255-262.

[51] B. Szegedy, 'An information theoretic approach to Sidorenko's conjecture', Preprint, arXiv:1406.6738.

[52] E. Szemerédi, 'Regular partitions of graphs', in Problèmes combinatoires et théorie des graphes (Colloq. Internat. CNRS, Univ. Orsay, Orsay, 1976), Colloq. Internat. CNRS, 260 (CNRS, Paris, 1978), 399-401.

[53] A. Thomason, 'Pseudorandom graphs', in Random Graphs '85 (Poznań, 1985), NorthHolland Mathematics Studies, 144 (North-Holland, Amsterdam, 1987), 307-331.

[54] A. Thomason, 'Random graphs, strongly regular graphs and pseudorandom graphs', in Surveys in Combinatorics 1987 (New Cross, 1987), London Mathematics Society Lecture Note Series, 123 (Cambridge University Press, Cambridge, 1987), 173-195.

[55] H. Towsner, ' $\sigma$-algebras for quasirandom hypergraphs', Random Structures Algorithms 50(1) (2017), 114-139.

[56] Richard M. Wilson, 'Cyclotomy and difference families in elementary abelian groups', J. Number Theory 4 (1972), 17-47.

[57] Raphael Yuster, 'Quasi-randomness is determined by the distribution of copies of a fixed graph in equicardinal large sets', Combinatorica 30(2) (2010), 239-246. 